PROCEEDINGS OF THE

AMERICAN MATHEMATICAL SOCIETY

Volume 139, Number 5, May 2011, Pages 1831-1839

S 0002-9939(2010)10588-9

Article electronically published on October 7, 2010

\title{
ON THE THREE-DIMENSIONAL BLASCHKE-LEBESGUE PROBLEM
}

\author{
HENRI ANCIAUX AND BRENDAN GUILFOYLE
}

(Communicated by Jon G. Wolfson)

\begin{abstract}
The width of a closed convex subset of $n$-dimensional Euclidean space is the distance between two parallel supporting hyperplanes. The Blaschke-Lebesgue problem consists of minimizing the volume in the class of convex sets of fixed constant width and is still open in dimension $n \geq 3$. In this paper we describe a necessary condition that the minimizer of the Blaschke-Lebesgue must satisfy in dimension $n=3$ : we prove that the smooth components of the boundary of the minimizer have their smaller principal curvature constant and therefore are either spherical caps or pieces of tubes (canal surfaces).
\end{abstract}

\section{INTRODUCTION}

The width of a convex body $B$ in $n$-dimensional Euclidean space in the direction $\vec{u}$ is the distance between the two supporting hyperplanes of $B$ which are orthogonal to $\vec{u}$. When this distance is independent of $\vec{u}, B$ is said to have constant width. The ratio $\mathcal{I}(B)$ of the volume of a constant width body to the volume of the ball of the same width is a homothety invariant, as is the isoperimetric ratio. Moreover, the maximum of $\mathcal{I}(B)$, like the minimum of the isoperimetric ratio, is attained by round spheres. However, while the isoperimetric ratio is not bounded from above, the infimum of $\mathcal{I}$ is strictly positive, since compactness properties of the space of convex sets ensures the existence of a minimizer. It is known by the work of Blaschke and Lebesgue that the Reuleaux triangle, obtained by taking the intersection of three discs centered at the vertices of an equilateral triangle, minimizes $\mathcal{I}$ in dimension $n=2$. The determination of the minimizer of $\mathcal{I}$ in any dimension is referred to as the Blaschke-Lebesgue problem.

Recently several simpler solutions of the problem in dimension 2 have been given (see $\mathrm{Ba}, \mathrm{Ha}]$ ). However the Blaschke-Lebesgue problem in dimension $n \geq 3$ appears to be very difficult to solve and remains open. A crucial step in solving the Blaschke-Lebesgue problem in dimension $n=2$ consists of proving that the boundary of the minimizer is made up of arcs of circles of radii equal to the width, and hence the smooth parts of the boundary have constant curvature.

In this paper we give a property of the minimizer of the Blaschke-Lebesgue in dimension $n=3$ which generalizes the constant curvature condition observed in

Received by the editors June 19, 2009 and, in revised form, August 19, 2009, August 20, 2009, and May 17, 2010.

2010 Mathematics Subject Classification. Primary 52A40, 52A15.

The first author was supported by Science Foundation Ireland (Research Frontiers Program).

(C)2010 American Mathematical Society 1831

Reverts to public domain 28 years from publication 
dimension $n=2$ (here and in the following, "smooth" means "continuously twice differentiable"):

Main Theorem. Let $B$ be a local minimizer of the Blaschke-Lebesgue problem in $\mathbb{R}^{3}$ with constant width $2 w$. Then the smooth parts of its boundary have their smaller principal curvature constant and equal to $1 / 2 w$.

It is easily seen that the boundary of a constant width body in $\mathbb{R}^{3}$ cannot be made up of only spherical caps, so the minimizer of the Blaschke-Lebesgue problem must have a more complicated geometry. On the other hand, K. Shiohama and R. Takagi proved in $[\mathrm{ST}$ that a non-spherical surface with one constant principal curvature must be a canal surface, i.e. the envelope of a one-parameter family of spheres or, equivalently, a tube over a curve (i.e. the set of points which lie at a fixed distance from this curve). Thus the main theorem implies the following:

Corollary. Let $B$ be a local minimizer of the Blaschke-Lebesgue problem in $\mathbb{R}^{3}$ with constant width $2 w$. Then the smooth parts of its boundary are spherical caps or pieces of tubes, both of them with radius equal to the width $2 w$ of $B$.

We observe that the constant width body having the best known ratio $\mathcal{I}$, Meissner body ( $\mathrm{CG}, \mathrm{GK}, \mathrm{Ba})$, satisfies this criteria: it is made up of four spherical caps centered at the vertices of a tetrahedron and three tubes over three arcs of circles. Therefore we cannot discard the possibility that it is the solution of the Blaschke-Lebesgue problem, although one might expect the minimizer to have tetrahedral symmetry. Another interesting constant width body is the one obtained by rotation of the Reuleaux triangle about an axis of symmetry. It is known that the latter minimizes the ratio $\mathcal{I}$ among constant width bodies with rotational symmetry (see $\mathrm{CCG}, \mathrm{AG}$ ). It is also interesting to note that this body satisfies our criteria as well: one part of its boundary is a spherical cap, and the other one is a tube over an arc of a circle. However, it has a bigger ratio $\mathcal{I}$ than Meissner's, which in particular proves that the solution of the Blaschke-Lebesgue problem does not have rotational symmetry.

In the light of our result, the most difficult issue to address seems to be that of regularity. We cannot exclude a priori that the boundary of the minimizer of the Blaschke-Lebesgue problem is singular everywhere, and the traditional techniques of regularity theory (e.g. those used for harmonic maps or minimal surfaces) do not seem to apply here. On the other hand, assuming that the minimizer is made up of a finite number of smooth parts, our result reduces the problem to a kind of combinatoric (though not easy) one: minimize the volume among the convex bodies whose boundaries are made up of spherical caps and pieces of tubes, all of them of the same radius.

As in $\mathrm{Ba}$, $\mathrm{Ha}$ and $\mathrm{AG}$, our proof is based on the analysis of the support function $s$ which characterizes a convex body $B$ of constant width $2 w$. The first point consists of evaluating the volume of $B$ and the area of its boundary in terms of $w$ and the function $h=s-w$ (Theorem 2). Our formula allows us in particular to easily prove the famous Blaschke formula, a functional relation between the volume, the area and the width of $B$, and to recover the fact that the ratio $\mathcal{I}$ is maximized by round spheres. A crucial point is then the following observation, stated in GK: flowing the boundary of a convex body along its inward unit normal vector field preserves the constant width condition as long as the evolving surface remains convex. Moreover, the ratio $\mathcal{I}$ decreases along the flow, so the minimizer 
of $\mathcal{I}$ must occur at the latest time such that convexity holds and therefore must be singular. This issue is easily controlled since the function $h$ is invariant along the normal flow, while the width $2 w$ decreases linearly. Thus, there exists a positive number $w_{0}(h)$ such that for any $w \geq w_{0}(h)$, the function $s=h+w$ is the support function of some convex body of constant width $2 w$. Hence, we can restrict the minimization process to the class of support functions of the form $s=h+w_{0}(h)$, while all the necessary information is carried by the function $h$. The main theorem is then obtained as follows: assuming that the smaller principal curvature is not constant on some smooth part of the boundary, we compute the second variation of $\mathcal{I}$ for a suitable local deformation of $h$ to get a contradiction.

The authors wish to thank the referee for correcting the statement of Theorem 3 .

\section{The GEOMETRY OF CONSTANT Width BODIES}

Let $B$ be a convex body in $\mathbb{R}^{n}$ and denote by $s$ its support function; i.e. $s(u)=$ $\sup _{x \in B}\langle u, x\rangle, \forall u \in \mathbb{S}^{n-1}$. Then the width $w(u)$ of $B$ in the direction $u$ is related to the support function by the following formula:

$$
2 w(u)=s(u)+s(-u)
$$

where $-u$ is the antipodal point of $u$ in $\mathbb{S}^{n-1}$. It is known (see [Ho, Ba] that if $B$ has constant width it must be strictly convex; moreover it is proven in $[\mathrm{Ho}$ that the support function $s$ of a constant width body is $C^{1,1}$; i.e. it admits first derivatives which are Lipschtiz continuous. By the Rademacher theorem, it follows that the second derivatives are well defined almost everywhere and bounded. This fact will be important later on, since the geometry of the boundary of $B$ will be expressed in terms of the Hessian of $h$.

If $B$ is a strictly convex body in $\mathbb{R}^{n}$ whose support function $s$ belongs to $C^{1,1}$, the map

$$
\begin{array}{rlc}
f: \mathbb{S}^{n-1} & \rightarrow & \mathbb{R}^{n}, \\
u & \mapsto & s(u) \cdot u+\nabla s(u)
\end{array}
$$

is a parametrization of its boundary and $u$ is the Gauss map of $\partial B$.

Given an arbitrary strictly convex body $B$, let $w \in \mathbb{R}$ be the mean of its support function $s$ on $\mathbb{S}^{n-1}$,

$$
w:=\frac{\int_{\mathbb{S}^{n-1}} s(u) d A}{\int_{\mathbb{S}^{n-1}} d A},
$$

where $d A$ denotes the canonical volume form on $\mathbb{S}^{n-1}$, and introduce the zero mean map $h:=s-w$. Then $B$ has constant width $2 w$ if and only if the function $h$ is odd, i.e.

$$
h(u)+h(-u)=0
$$

The following inequality will be crucial for us:

Proposition 1 (Wirtinger inequality). Let $h \in C^{1,1}\left(\mathbb{S}^{n-1}\right)$ with vanishing mean and let $d A$ be the volume element on $\mathbb{S}^{n-1}$. Then

$$
\mathcal{E}(h):=\int_{\mathbb{S}^{n-1}}\left(\frac{1}{n-1}|\nabla h|^{2}-h^{2}\right) d A \geq 0,
$$

with equality if $h$ is a first eigenfunction of the Laplacian on the sphere $\mathbb{S}^{n-1}$. 
This result is easily proved once the theory of spherical harmonics, generalizing Fourier analysis to higher dimension, is developed (see [GW], p. 1288).

From now on we restrict ourselves to the case of dimension 3. Our first step consists of expressing the local geometry of the boundary of a convex body $B$ in terms of the data $(h, w)$. We recall that the Hessian of $h$ is the symmetric tensor defined by $\operatorname{Hess}(h)(X, Y)=\left\langle\nabla_{X} \nabla h, Y\right\rangle$, where $\nabla$ denotes the Levi-Civita connection of the round metric of $\mathbb{S}^{2}$. The two invariants of Hess $(h)$ are its trace, which is the well known Laplace-Beltrami operator $\Delta$, and its determinant, which we shall denote in the following by $H(h)$.

Theorem 1. The area element of $\partial B$, denoted by $d \bar{A}$, is given by

$$
d \bar{A}=\left(w^{2}+\alpha w+\beta\right) d A
$$

where we set

$$
\alpha:=2 h+\Delta h \quad \text { and } \quad \beta:=h^{2}+h \Delta h+H(h) .
$$

Moreover, its principal curvatures $k_{1}$ and $k_{2}$, whenever they exist, take the following form:

$$
k_{1,2}=\frac{2 w+\alpha \pm \sqrt{\alpha^{2}-4 \beta}}{2\left(w^{2}+\alpha w+\beta\right)} .
$$

In the case where $B$ has constant width, we deduce the following formulas for its volume $\mathcal{V}(B)$ and the area of its boundary $\mathcal{A}(\partial B)$ :

Theorem 2. Let $B$ be a convex body of constant width $2 w$ in $\mathbb{R}^{3}$. Then

$$
\begin{aligned}
& \mathcal{V}(B)=\frac{4 \pi}{3} w^{3}-w \mathcal{E}(h), \\
& \mathcal{A}(\partial B)=4 \pi w^{2}-\mathcal{E}(h) .
\end{aligned}
$$

This allows us to recover the famous Blaschke formula, a functional relation between the volume, the area and the width:

Corollary 1 (Blaschke formula). Let $B$ be a convex body in $\mathbb{R}^{3}$ of constant width $2 w$. Then

$$
\mathcal{V}(B)=w \mathcal{A}(\partial B)-\frac{8}{3} \pi w^{3} .
$$

The proofs of Theorems 1 and 2 are postponed until the end of the paper (Sections 4 and 5).

\section{The Blaschke-Lebesgue problem}

Let $B$ be a convex body of constant width $2 w$ and denote by $B_{w}$ the round ball of radius $w$. Introduce the ratio

$$
\mathcal{I}(B)=\frac{\mathcal{V}(B)}{\mathcal{V}\left(B_{w}\right)}=\frac{\mathcal{V}(B)}{4 \pi w^{3} / 3} .
$$

By Theorem 2, we have

$$
\mathcal{I}(B)=\mathcal{I}(h, w)=1-\frac{\mathcal{E}(h)}{4 \pi w^{2} / 3} .
$$

It follows from the Wirtinger inequality that the ratio $\mathcal{I}(B)$ is less than or equal to 1 and that equality is attained when $h$ is a first eigenfunction of the Laplacian, as it is in the case of balls $B=B_{w}$. Moreover, for a given $h, \mathcal{I}$ increases with respect to $w$. Hence it reaches its minimum at the lowest value of $w$ such that $h+w=s$ is 
the support function of a convex body; we define $w_{0}(h)$ to be this crucial quantity. Increasing (resp. decreasing) the value of $w$ corresponds geometrically to flowing the boundary of $B$ parallel to itself, i.e. along its outward (resp. inward) normal vector. Therefore the map $h$ corresponds to a one-parameter family of parallel surfaces, labelled by the parameter $w \in\left[w_{0}(h), \infty\right)$. The inward normal flow can be continued as long as the surface is smooth. By Theorem 1, this is equivalent to the fact that the area element $d \bar{A}$ is strictly positive. Hence, we deduce an explicit expression for $w_{0}(h)$,

$$
w_{0}(h)=\inf \left\{w \in \mathbb{R}^{+} \mid w^{2}+\alpha w+\beta>0 \text { a.e. on } \mathbb{S}^{2}\right\},
$$

and the convex body $B$ corresponding to $s=h+w_{0}(h)$ is always singular.

Remark 1. One can check that $w_{0}(h)=\|W(h)\|_{L^{\infty}\left(\mathbb{S}^{2}\right)}$, where

$$
W(h)(u):=\frac{-\alpha+\sqrt{\alpha^{2}-\beta}}{2} .
$$

The directions $u$ of $\mathbb{S}^{2}$ where the area element vanishes correspond precisely to points $f(u)$ of the boundary which are singular. The next theorem shows that in the smooth parts of a local minimizer of $\mathcal{I}$, such a situation actually occurs for every pair of antipodal directions $(u,-u)$. We point out that this result is roughly equivalent to one of the main results of [BLO] (Theorem 5).

Theorem 3. Let $\left(h, w_{0}(h)\right)$ be a local minimizer of $\mathcal{I}(h, w)$ and let $U$ be an open subset of $\mathbb{S}^{2}$ where $h$ is smooth. Then for every point $u$ of $U$, the area element $d \bar{A}$ vanishes at one of the points $u$ and $-u$.

Proof. We proceed by contradiction, assuming that there is an open subset $U$ of $\mathbb{S}^{2}$ where $h$ is smooth and such that $\left(w_{0}(h)\right)^{2}+\alpha w_{0}(h)+\beta>0$ in $U \cup(-U)$. Consider a smooth map $v$ such that $v(u)+v(-u)=0, \forall u \in \mathbb{S}^{2}$ and whose support is contained in $U \cup(-U)$, and define the deformation $h^{\epsilon}:=h+\epsilon v$ of $h$. For small $\epsilon$,

$$
w_{0}\left(h^{\epsilon}\right)=w_{0}(h) ;
$$

hence

$$
\frac{\mathcal{E}\left(h^{\epsilon}\right)}{w_{0}^{2}\left(h^{\epsilon}\right)}=\frac{\mathcal{E}(h)}{w_{0}^{2}(h)}+\epsilon \frac{\delta \mathcal{E}(h, v)}{w_{0}^{2}(h)}+\frac{\epsilon^{2}}{2} \frac{\delta^{2} \mathcal{E}(h, v)}{w_{0}^{2}(h)}+o\left(\epsilon^{2}\right) .
$$

As $h$ is a minimizer of $\mathcal{I}$, and thus a maximizer of $\mathcal{E}(h) / w_{0}^{2}(h)$, we must have both $\delta \mathcal{E}(h, v)=0$ and $\delta^{2} \mathcal{E}(h, v) \leq 0$. On the other hand the functional $\mathcal{E}$ is quadratic so that $\delta^{2} \mathcal{E}(h, v)=\mathcal{E}(v)$, which is positive by the Wirtinger inequality (Proposition 11). Finally, the support of $v$ being contained in $U \cup(-U), v$ cannot be an eigenfunction of the Laplacian, and we obtain the required contradiction.

\section{Proof of the MAin theorem}

We are now in position to prove our main result: assume that $B$ is a local minimizer of $\mathcal{I}(B)$ and let $h$ be the associated map. For the sake of brevity we set $\tilde{w}:=w_{0}(h)$ in the following. Let $U$ be an open subset of $\mathbb{S}^{2}$ such that $f(U)$ is a smooth part of $\partial B$. In particular, by Theorem 1. $\tilde{w}^{2}+\alpha \tilde{w}+\beta>0$ on $U$. Hence by Theorem 3, $\tilde{w}^{2}+\alpha(u) \tilde{w}+\beta(u)=0, \forall u \in-U$. Since $\alpha$ is odd and $\beta$ is even, it 
follows that $\tilde{w}^{2}-\alpha(u) \tilde{w}+\beta(u)=0, \forall u \in U$. Consequently, by Theorem 1,

$$
\begin{aligned}
k_{1,2} & =\frac{2 \tilde{w}+\alpha \pm \sqrt{\alpha^{2}-4 \beta}}{2\left(\tilde{w}^{2}+\alpha \tilde{w}+\beta\right)}=\frac{2 \tilde{w}+\alpha \pm \sqrt{\alpha^{2}-4 \alpha \tilde{w}+4 \tilde{w}^{2}}}{2\left(\tilde{w}^{2}+\alpha \tilde{w}+\left(\alpha \tilde{w}-\tilde{w}^{2}\right)\right)} \\
& =\frac{2 \tilde{w}+\alpha \pm|\alpha-2 \tilde{w}|}{4 \alpha \tilde{w}},
\end{aligned}
$$

so that $k_{1}=\frac{1}{\alpha}$ and $k_{2}=\frac{1}{2 \tilde{w}}$. Hence the principal curvature $k_{2}$ is constant on $U$ and equal to the inverse of the width $2 \tilde{w}$ of $B$. Finally, since $\alpha(u) \geq 0$, we have

$$
2 \tilde{w}=-\alpha(-u)+\sqrt{\alpha^{2}(-u)-4 \beta(-u)}=\alpha(u)+\sqrt{\alpha^{2}(u)-4 \beta(u)} \geq \alpha,
$$

so $\frac{1}{2 \tilde{w}} \leq \frac{1}{\alpha}$; that is, $k_{2}$ is the smaller curvature, and the proof is complete.

\section{The local geometry of $\partial B$ (proof of Theorem 1)}

Let $(x, y)(u)$ be an isothermic coordinate chart from a dense subset of $\mathbb{S}^{2}$ into an open subset $U$ of $\mathbb{R}^{2}$ (for example the stereographic projection onto $U=\mathbb{R}^{2}$ ), and denote by $e^{r}$ the conformal factor, i.e. $e^{r}=\left|\partial_{x}\right|=\left|\partial_{y}\right|$. In particular the area element is given by $d A=e^{r} d x d y$. The coefficients of the Hessian of $h$ in the coordinates $(x, y)$ are

$$
\begin{aligned}
a & :=e^{-2 r}\left\langle\nabla_{\partial_{x}} \nabla h, \partial_{x}\right\rangle, \\
b & :=e^{-2 r}\left\langle\nabla_{\partial_{x}} \nabla h, \partial_{y}\right\rangle=e^{-2 r}\left\langle\nabla_{\partial_{y}} \nabla h, \partial_{x}\right\rangle, \\
c & :=e^{-2 r}\left\langle\nabla_{\partial_{y}} \nabla h, \partial_{y}\right\rangle .
\end{aligned}
$$

We recall that the boundary of $\partial B$ is parametrized by $f(u)=s(u) u+\nabla s(u)=$ $s(u) u+\nabla h(u)$. In order to compute the first derivatives of $f$, we use the Gauss formula of the embedding of the sphere $\mathbb{S}^{2}$ in $\mathbb{R}^{3}$, which relates the flat connection $D$ of $\mathbb{R}^{3}$ to the Levi-Civita connection $\nabla$ on the sphere:

$$
\left(D_{X} Y\right)(u)=\left(\nabla_{X} Y\right)(u)-\langle X, Y\rangle u \text {. }
$$

It follows that

$$
f_{x}=s \partial_{x}+\nabla_{\partial_{x}} \nabla h=(s+a) \partial_{x}+b \partial_{y}
$$

and

$$
f_{y}=s \partial_{y}+\nabla_{\partial_{y}} \nabla h=b \partial_{x}+(s+c) \partial_{y} .
$$

The trace $a+c=\Delta h$ and the determinant $H(h):=a c-b^{2}$ of the Hessian matrix of $h$ are intrinsic quantities; i.e. they depend only on the metric on $\mathbb{S}^{2}$ and not on the choice of coordinates.

We then compute the coefficients of the first fundamental form of the immersion $f$ :

$$
\begin{aligned}
& E:=\left\langle f_{x}, f_{x}\right\rangle=\left((s+a)^{2}+b^{2}\right) e^{2 r}, \quad F:=\left\langle f_{x}, f_{y}\right\rangle=(2 s+a+c) b e^{2 r}, \\
& G:=\left\langle f_{y}, f_{y}\right\rangle=\left((s+c)^{2}+b^{2}\right) e^{2 r} .
\end{aligned}
$$

It follows that

$$
\begin{aligned}
& \sqrt{E G-F^{2}} e^{-2 r}=\left(\left((s+a)^{2}+b^{2}\right)\left((s+c)^{2}+b^{2}\right)-4(s+a+s+c)^{2} b^{2}\right)^{1 / 2} \\
& =\left(s^{2}+s(a+c)+a c-b^{2}\right)=\left(w^{2}+(2 h+a+c) w+h^{2}+(a+c) h+a c-b^{2}\right),
\end{aligned}
$$


and we deduce the first part of Theorem 1:

$$
\begin{aligned}
d \bar{A} & =\sqrt{E G-F^{2}} d x d y=\sqrt{E G-F^{2}} e^{-2 r} d A \\
& =\left(\left(w^{2}+(2 h+\Delta h) w+h^{2}+h \Delta h+H(h)\right) d A .\right.
\end{aligned}
$$

Next we calculate the coefficients of the second fundamental form: since $N(u)=$ $u$, we have

$$
\begin{aligned}
l & :=\left\langle\partial_{x} N(u), f_{x}\right\rangle=\left\langle\partial_{x} u, f_{x}\right\rangle=e^{2 r}(s+a), \\
m & :=\left\langle\partial_{x} N(u), f_{y}\right\rangle=\left\langle\partial_{x} u, f_{y}\right\rangle=e^{2 r} b, \\
n & :=\left\langle\partial_{y} N(u), f_{y}\right\rangle=\left\langle\partial_{y} u, f_{y}\right\rangle=e^{2 r}(s+c) .
\end{aligned}
$$

Thus

$$
\begin{aligned}
l G & +n E-2 m F \\
& =e^{4 r}\left((s+a)\left((s+c)^{2}+b^{2}\right)+(s+c)\left((s+a)^{2}+b^{2}\right)-2 b^{2}(2 s+a+c)\right) \\
& =e^{4 r}\left((s+a)(s+c)(2 s+a+c)-b^{2}(2 s+a+c)\right) \\
& =e^{4 r}\left(w^{2}+\alpha w+\beta\right)(2 w+\alpha)
\end{aligned}
$$

and

$$
\ln -m^{2}=e^{4 r}\left((s+c)(s+a)-b^{2}\right)=e^{4 r}\left(w^{2}+\alpha w+\beta\right) .
$$

Thus, at a point $f(u)$ where $d \bar{A}$ does not vanish, the mean curvature and the Gaussian curvature of $\partial B$ are given by

$$
2 H=\frac{l G+n E-2 m F}{E G-F^{2}}=\frac{2 w+\alpha}{w^{2}+\alpha w+\beta}
$$

and

$$
K=\frac{\ln -m^{2}}{E G-F^{2}}=\frac{1}{w^{2}+\alpha w+\beta},
$$

so that

$$
H^{2}-K=\frac{(2 w+\alpha)^{2}-4\left(w^{2}+\alpha w+\beta\right)}{4\left(w^{2}+\alpha w+\beta\right)^{2}}=\frac{\alpha^{2}-4 \beta}{4\left(w^{2}+\alpha w+\beta\right)^{2}} .
$$

Hence the principal curvatures $k_{1}$ and $k_{2}$ of the immersion at the point $f(u)$ are

$$
k_{1,2}=H \pm \sqrt{H^{2}-K}=\frac{2 w+\alpha \pm \sqrt{\alpha^{2}-4 \beta}}{2\left(w^{2}+\alpha w+\beta\right)} .
$$

\section{Volume and area of $\partial B$ (proof of Theorem 2)}

The only tricky part of the proof is the following lemma:

Lemma 1. If $B$ has constant width, then

$$
\int_{\mathbb{S}^{2}} H(h) d A=\frac{1}{2} \int_{\mathbb{S}^{2}}|\nabla h|^{2} d A .
$$

Proof. Denoting the complex structure on $\mathbb{S}^{2}$ by $j$, we have $j \partial_{x}=\partial_{y}, j \partial_{y}=-\partial_{x}$. The proof is based on the following formula for the curvature tensor on the sphere:

$$
\langle R(X, Y) Z, W\rangle=\langle X, Z\rangle\langle Y, W\rangle-\langle Y, Z\rangle\langle X, W\rangle
$$


with $X=\partial_{x}, Y=\partial_{y}, Z=\nabla h, W=j \nabla h$. On the one hand,

$$
\begin{aligned}
\int_{U} & (\langle X, Z\rangle\langle Y, W\rangle-\langle Y, Z\rangle\langle X, W\rangle) d x d y \\
= & \int_{U}\left(\left\langle\partial_{x}, \nabla h\right\rangle\left\langle j \partial_{x}, j \nabla h\right\rangle-\left\langle\partial_{y}, \nabla h\right\rangle\left\langle\partial_{y}, j \nabla h\right\rangle\right) d x d y \\
= & \int_{U}\left(h_{x}^{2}+h_{y}^{2}\right) d x d y=\int_{\mathbb{S}^{2}}|\nabla h|^{2} d A .
\end{aligned}
$$

On the other hand, using the fact that $j$ is parallel, i.e. $\nabla_{X} j Y=j \nabla_{X} Y$, we have

$$
\begin{aligned}
\int_{U} & \left\langle R\left(\partial_{x}, \partial_{y}\right) \nabla h, j \nabla h\right\rangle d x d y \\
& =\int_{U}\left(\left\langle\nabla_{\partial_{y}} \nabla_{\partial_{x}} \nabla h, j \nabla h\right\rangle-\left\langle\nabla_{\partial_{x}} \nabla_{\partial_{y}} \nabla h, j \nabla h\right\rangle\right) d x d y \\
& =\int_{U}\left(-\left\langle\nabla_{\partial_{x}} \nabla h, \nabla_{\partial_{y}} j \nabla h\right\rangle+\left\langle\nabla_{\partial_{y}} \nabla h, \nabla_{\partial_{x}} j \nabla h\right\rangle\right) d x d y \\
& =\int_{U}\left(-\left\langle\nabla_{\partial_{x}} \nabla h, j \nabla_{\partial_{y}} \nabla h\right\rangle+\left\langle\nabla_{\partial_{y}} \nabla h, j \nabla_{\partial_{x}} \nabla h\right\rangle\right) d x d y \\
& \left.=2 \int_{U}\left\langle\nabla_{\partial_{y}} \nabla h, j \nabla_{\partial_{x}} \nabla h\right\rangle\right) d x d y \\
& =2 \int_{U}\left\langle\left(b \partial_{x}+c \partial_{y}\right),\left(-b \partial_{x}+a \partial_{y}\right)\right\rangle d x d y \\
& =2 \int_{U}\left(a c-b^{2}\right) e^{2 r} d x d y=2 \int_{\mathbb{S}^{2}} H(h) d A
\end{aligned}
$$

hence the proof of the lemma is complete.

In order to calculate the volume of $B$, we use the divergence theorem. Recalling that $u$ is the unit outward normal vector of the smooth parts of $\partial B$ and that $f(u)=s(u) u+\nabla h(u)$, we have

$$
\begin{aligned}
\mathcal{V}(B)= & \frac{1}{3} \int_{\mathbb{S}^{2}}\langle f(u), u\rangle d \bar{A}=\frac{1}{3} \int_{\mathbb{S}^{2}} s(u) d \bar{A} \\
= & \frac{1}{3} \int_{\mathbb{S}^{2}}(h+w)\left(w^{2}+\alpha w+\beta\right) d A \\
= & \frac{w^{3}}{3} \int_{\mathbb{S}^{2}} d A+\frac{w^{2}}{3} \int_{\mathbb{S}^{2}}(3 h+\Delta h) d A+\frac{w}{3} \int_{\mathbb{S}^{2}}\left(3 h^{2}+2 h \Delta h+H(h)\right) d A \\
& +\frac{1}{3} \int_{\mathbb{S}^{2}}\left(h^{3}+h^{2} \Delta h+h H(h)\right) d A .
\end{aligned}
$$

Since $h$ has zero mean, the coefficient of $w^{2}$ vanishes. Moreover, the constant width condition, i.e. the oddness of $h$, implies that all the cubic expressions of $h$ and its second derivatives are odd and hence have zero mean. Thus the constant term 
vanishes. Finally, using the divergence theorem and Lemma 1 we obtain

$$
\begin{aligned}
\mathcal{V}(B) & =\frac{w^{3}}{3} \int_{\mathbb{S}^{2}} d A+w \int_{\mathbb{S}^{2}}\left(h^{2}+\left(-\frac{2}{3}+\frac{1}{6}\right)|\nabla h|^{2}\right) d A \\
& =\frac{4 \pi w^{3}}{3}-w \int_{\mathbb{S}^{2}}\left(\frac{1}{2}|\nabla h|^{2}-h^{2}\right) d A \\
& =\frac{4 \pi w^{3}}{3}-w \mathcal{E}(h),
\end{aligned}
$$

which is the required formula.

The computation of the area of $\partial B$ uses Lemma 1 as well and is straightforward:

$$
\begin{aligned}
\mathcal{A}(\partial B) & =\int_{\partial B} d \bar{A}=\int_{\mathbb{S}^{2}}\left(w^{2}+(2 h+\Delta h) w+\left(h^{2}+h \Delta h+H(h)\right)\right) d A \\
& =w^{2} A\left(\mathbb{S}^{2}\right)+\int_{\mathbb{S}^{2}} h^{2} d A+\int_{\mathbb{S}^{2}}|\nabla h|^{2} d A-\frac{1}{2} \int_{\mathbb{S}^{2}}|\nabla h|^{2} d A \\
& =4 \pi^{2}-\mathcal{E}(h) .
\end{aligned}
$$

\section{REFERENCES}

[AG] H. Anciaux, N. Georgiou, The Blaschke-Lebesgue problem for constant width bodies of revolution, arXiv:0903.4284.

[Ba] T. Bayen, PhD Dissertation, Optimisation de formes dans la classe des corps de largeur constante et des rotors, Université Pierre et Marie Curie - Paris 6, 2007.

[BLO] T. Bayen, T. Lachand-Robert, E. Oudet, Analytic parametrizations and volume mimization of three dimensional bodies of constant width, Arch. Ration. Mech. Anal. 186 (2007), no. 2, 225-249. MR2342202 (2008f:52005)

[CCG] S. Campi, A. Colesanti, P. Gronchi, Minimum problems for volumes of convex bodies, in Partial differential equations and applications, Vol. 177 of Lecture Notes in Pure and Appl. Math., Dekker, New York, 1996, 43-55. MR.1371579 (96j:52012)

[CG] G.D. Chakerian, H. Groemer, Convex bodies of constant width, in Convexity and its applications (Ed. P. Gruber and J. Wills), Birkhäuser, Basel, 1983, 49-96. MR731106 (85f:52001)

[GK] B. Guilfoyle, W. Klingenberg On $C^{2}$-smooth surfaces of constant width, Tbilisi Math. Journal 1 (2009), 1-17. MR2574869

[GW] P.-M. Gruber, J.-M. Wills, Handbook of convex geometry, North-Holland, Amsterdam, 1993. MR 1242973 (94e:52001)

[Ha] E. Harrell, II, A direct proof of a theorem of Blaschke and Lebesgue, J. Geom. Anal. 12 (2002), no. 1, 81-88. MR1881292 (2002k:52009)

[Ho] R. Howard, Convex bodies of constant width and constant brightness, Adv. Math. 204 (2006), no. 1, 241-261. MR2233133 (2007f:52004)

[ST] K. Shiohama, R. Takagi, A characterization of a standard torus in $E^{3}$, J. of Diff. Geom. 4 (1970), 477-485. MR0276906 (43:2646)

Universidade de São Paulo, ime, Bloco A, 1010 Rua do Matão, Cidade Universitária, 05508-090 SÃo PaUlo, Brazil

E-mail address: henri.anciaux@gmail.com

Department of Mathematics and Computing, Institute of Technology, Tralee, County Kerry, Ireland

E-mail address: brendan.guilfoyle@ittralee.ie 